\title{
Editorial
}

\section{Is there a role of Eastern Europe in cancer biomarkers for personalized cancer medicine?}

\author{
I. Berindan-Neagoe ${ }^{\mathrm{a}, *}$ and A. Paradiso ${ }^{\mathrm{b}}$ \\ ${ }^{\mathrm{a}}$ The University of Medicine and Pharmacy Iuliu Hatieganu, Cluj, Napoca, Romania \\ ${ }^{\mathrm{b}}$ National Cancer Research Center, Istituto Tumori G Paolo II, IRCCS, Bari, Italy
}

Personalized medicine (PM) is considered the medicine of the future, presenting the first concept in creating a patient-specific tailored therapy. With this new approach, the new "cascade" of methods and applications developed in molecular biology over the past 10 years will play a significant role. Different options in the genomic and proteomic characterization of each patient has presented new challenges in cancer therapy.

Are we going to use different treatments for diseases displaying similarities? Are we going to use similar treatments for patients with different cancers but similar molecular profiles?

Personalized medicine can identify those patients who are the most suitable for a specific drug or medication. However, the context matters as well, as it helps to identify resistant diseases and patients who will not respond to the therapy. Consequently, patients will suffer no side effects with full therapy effectiveness.

Finally, the purpose of this new concept of personalized medicine is to develop therapies that will significantly improve patients' outcomes.

Why Eastern Europe?

Although part of Europe, Romania faces problems due to financial constraints. One of the major disadvantages is diagnosing cancer cases at advanced stages, including cervicalvica cancer (already eradicated in US or Western Europe), as well as cancers of the breast, colon, prostate, bladder, ovary, thyroid, gastric and lungs. One of the major challenges in oncology in Eastern Europe will be early diagnosis, followed by an individualized, tailored care of cancer patients. We have to underline the major role of cancer biomarkers for personalized medicine if we consider as compulsory that Eastern Europe is a part of this future. Personalized medicine is based on the concept that cancer medicine is able to develop a number of technologies such as genomics, transcriptomics and proteomics to acquire data at molecular level about DNA, RNA and the proteins of the patients. In addition, PM would be capable of acquiring functional imaging modifications and identifying high risk patients. More research is is needed to focuson the identification and validation of cancer biomarkers from different molecules and structures: miRNA, long non-coding RNAs, or CpG epigenetic modifications. Specific approaches to molecular classification of cancer biomarkers like claudin-low breast cancer, Her2 positive breast cancer, basal-like breast cancer, hereditary gynecological cancers, BRCA1 and 2 mutations, miRnome profile, K-Ras, EGFR, BRAF V600, and bcr-abl mutations are just some well known biomarkers in cancer.

The Oncology Institute Ion Chiricuta in Cluj Napoca, Romania is the most important cancer unit in Transylvania and perhaps the most important in Romania. However, the Institute still needs to open itsd doors to outstanding researchers and practitioners in oncology.

Scientists who have contributed their research at several conferences and workshops with European societies have created an opening for future international cooperation. 
This special issue, produced with the strategic collaboration of the National Cancer Research Center of Bari, Italy, and with the support of European Organization of Cancer Institutes (OECI), provides us with an important opportunity to present to the professional community our research and interest in the field.

How is Romanian personalized medicine evolving in the European environment? The strategic instrument to develop the scientific integration of Europe and to strengthen its international outreach has been clearly indicated by the European Commission in the European Strategy Forum on Research Infrastructures, ESFRI. The mission of ESFRI is to support a coherent and strategy-led approach to policy-making on research infrastructures in Europe and to facilitate multilateral initiatives leading to the better use and development of research infrastructures, at the EU and international level. Currently, a dozen different biological and medical sciences (BMS) research infrastructures are prioritized in the second update of the ESFRI Roadmap in 2010.

In the field of personalized medicine/biomarker development, some of these infrastructures are of crucial relevance:

- European advanced translational research infrastructure in medicine (EATRIS),

- European clinical research infrastructures network (ECRIN),

- European life science infrastructure for biological information (ELIXIR),
- European biomedical imaging infrastructure (Euro-Bioimaging), and

- Biobanking and Biomolecular Research Infrastructure (BBMRI).

According to the ESFRI, scientists from all over Europe will benefit from technologies, know-how, and services developed by all these infrastructures. This includes sharing biospecimens (BBMRI) and utilizing high-capacity computing and data storage solutions (ELIXIR) required for dealing with massive genomics, transcriptomics, metabolomics, and imaging data derived from human biological samples, thus collaborating to Biomarker discovery/validation (EATRIS) and innovative clinical trials (ECRIN).

To accomplish this goal, joint grant applications for improving the interoperability of data management between BMS research infrastructures and different countries have been already submitted to specific European structures. The development of coordinated strategies for education and training in the life sciences is a needed joint activity of all BMS research infrastructures.

In conclusion, if Romania is to remain at the rapidly evolving forefront of science and technology, there is a strong need to participate in the ESFRI Roadmap, actively participating in research infrastructures that are closer to Romania's medical and scientific interests. 